Research Article

\title{
Serum IL-5 and IFN- $\gamma$ Are Novel Predictive Biomarkers for Anti-PD-1 Treatment in NSCLC and GC Patients
}

\author{
Qiu Zhao, ${ }^{1}$ Yanzhi Bi, ${ }^{1}$ Huiting Sun, ${ }^{2}$ and Min Xiao $\mathbb{D}^{1}$ \\ ${ }^{1}$ Department of Oncology, Changzhou Tumor Hospital Affiliated to Soochow University, Changzhou 213000, China \\ ${ }^{2}$ Changzhou Second People's Hospital, Changzhou 213000, China
}

Correspondence should be addressed to Min Xiao; zkh135790@163.com

Received 23 January 2021; Accepted 20 May 2021; Published 19 June 2021

Academic Editor: Alvaro González

Copyright ( 2021 Qiu Zhao et al. This is an open access article distributed under the Creative Commons Attribution License, which permits unrestricted use, distribution, and reproduction in any medium, provided the original work is properly cited.

\begin{abstract}
Background. Because responses of patients with cancer to immunotherapy can vary in success, effective biomarkers are urgently needed for predicting clinical response with anti-PD-1 treatment. We aimed to evaluate the IL-5 and IFN- $\gamma$ level with the response of anti-PD-1 blockade in non-small-cell lung cancer (NSCLC) and gastric cancer (GC). Methods. Metastatic NSCLC and GC patients treated with anti-PD-1 monoclonal antibody were studied. Blood samples were taken before PD-1 McAb treatment, after the first cycle treatment, and during efficacy evaluation. The association between IL-5 and IFN- $\gamma$ levels and clinical response were analyzed by the nonparametric Wilcoxon matched-pairs ranked tests. The progression-free survival (PFS) time was obtained by imaging evaluation and telephone follow-up of all the patients. Kaplan-Meier and the log rank test were used to plot the survival curve. Results. IL-5 and IFN- $\gamma$ levels were detected in the peripheral blood of 40 NSCLC and 35 GC patients who have received anti-PD- 1 treatment. In effective group, IL-5 and IFN- $\gamma$ levels at best response points significantly decreased $(P<0.001)$ compared with pretherapeutic levels in NSCLC and GC patients with lymph node or distant metastasis. Compared with pretherapeutic levels, IL-5 and IFN- $\gamma$ levels largely increased as the tumor progresses $(P<0.01)$. Higher IL-5 and IFN- $\gamma$ levels before treatment indicated shorter progression-free survival in patients with NSCLC metastasis $(P=0.007, P=$ 0.0111). Moreover, their levels also accurately reflected the pseudoprogression of two NSCLC patients to anti-PD-1 treatment. Conclusions. Our results suggested that serum IL-5 and IFN- $\gamma$ levels could be an effective indicator for predicting clinical efficacy and survival with anti-PD-1 blockade in NSCLC and GC patients.
\end{abstract}

\section{Introduction}

Program Death 1(PD-1) expressed on T cells, B cells, and myeloid cells negatively regulates immune response. Deficiency of PD-1 leads to immune dysfunction and autoimmune disease [1]. Program Death Ligand 1(PD-L1), its primary ligand, belongs to $\mathrm{B} 7$ gene family and is variably expressed by lymphoid and nonlymphoid cells. The combination of PD-L1 and PD-1 leads to inhibition of T cellmediated cytokine secretion and lymphocyte recruitment [2]. When PD-L1 is widely expressed on tumor cells, it will provide an effective inhibitory tumor microenvironment [3]. PD-1/PD-L1 blocking antibodies provide a promising treatment for various cancers [4]. However, approximately $70 \%-80 \%$ of patients have failed to respond to this therapy, and excellent efficacy happened only with limited tumor types [5]. In addition, due to delayed kinetics and atypical response models, approximately $15 \%$ of patients have experienced pseudoprogression, which means that when images show tumor enlargement, the patients are clinically stable or improved [6].

These abnormal response patterns make it difficult to distinguish the effective patients from noneffective patients in the early stages of treatment. Therefore, it is essential to identify more accurate biomarkers to predict the response to PD$1 \mathrm{McAb}$ treatment. This can benefit patients who experience tumor shrinkage after the treatment.

Immune interferon (IFN), also known as IFN- $\gamma$, is an potent anticancer cytokine secreted from CTLs, NK cells, NKT cells, and $\gamma \delta$ T cells $[7,8]$. IFN- $\gamma$ plays an important role in the immune responses to infection and cancer. It can play its immunomodulatory effect by improving MHC- 
mediated antigen presentation, reinforcing type $1 \mathrm{~T}$ helper cell (Th1) responses, regulating leukocyte trafficking, facilitating Toll-like receptor signaling, and enhancing the antitumor and antimicrobial functions [9].

IL-5 is mainly produced by T helper-2 (Th2) lymphocytes and group 2 innate lymphoid cells (ILC2). It can increase antibody secretion through promoting the differentiation and growth of B cells and enhance the humoral immune response mediated by Th2 cells. Immunity to tumors is mainly governed by Th1-mediated cellular immunity. If Th1-Th2 drift occurs, it will lead to produce an immunosuppressive and development of cancer [10].

In our study, we investigated the correlation between serum IFN- $\gamma$ and IL-5 levels and the clinical response to anti-PD-1 mAbs in NSCLC and GC patients.

\section{Methods}

2.1. Patients and Response Criteria. From January 2017 to December 2019, a total of 75 patients with metastatic NSCLC and GC (57 males and 18 females) were enrolled in the Changzhou Tumor Hospital affiliated to Soochow University, including 40 patients with metastatic NSCLC and 35 patients with metastatic GC. The average age of the 75 patients was 65.5 years (range from 23 to 81 years). All patients gave informed consent before being enrolled in our study. Our study obtained ethical approval from the Ethics Committee of Changzhou Cancer Hospital. The experimental protocol reported in this paper is in accordance with World Medical Association Declaration of Helsinki on Ethical Principles for Medical Research Involving Human Subjects. Patients were enrolled with the following criteria: (1) clinical diagnosis of stage IV NSCLC or GC; (2) receive anti-PD-1 mAb treatment regardless of whether combinate with chemotherapy or molecular targeted therapy; (3) meet Eastern Cooperative Oncology Group (ECOG) physical status 0-2; and (4) good compliance and voluntary accept cytokine testing.

According to the RECIST 1.1 standard (11), the complete disappearance of the target lesions is evaluated as complete response (CR), a more than $30 \%$ reduction of total target lesions is evaluated as partial response (PR), a more than $20 \%$ increase of total target lesions is evaluated as progressive disease (PD), and a reduction less than $30 \%$ or an increase less than $20 \%$ in the sum of target lesions was assessed as stable disease (SD). Disease control rate (DCR) is equal to the ratio of $\mathrm{CR}+\mathrm{PR}+\mathrm{SD}$ cases to the total number of cases, and all of these have been maintained for more than 4 weeks. Two NSCLC patients were identified as pseudoprogression (after a first treatment cycle, a more than $20 \%$ increase of the tumor size that was assessed as a partial response by imaging system). Progression-free survival (PFS) is the time from the beginning of PD-1 monoclonal treatment to the first disease progression by imaging evaluation. The detailed clinical characteristics of the patients are described in Table 1 .

2.2. Sample Collection. Blood samples were collected from each patient before PD-1 McAb treatment, after the first cycle treatment, and in each subsequent radiographic evaluation.
TABLE 1: The clinicopathological parameters of patients.

\begin{tabular}{lcc}
\hline Parameters & NSCLC & Gastric cancer \\
\hline Gender, $n$ (\%) & $34(85)$ & $23(65.7)$ \\
$\quad$ Male & $6(15)$ & $12(34.3)$ \\
$\quad$ Female & & \\
Age (year) & $13(32.5)$ & $10(28.5)$ \\
$\quad \leqslant 60$ & $27(67.5)$ & $25(71.5)$ \\
$>60$ & $32(80)$ & $21(60)$ \\
Has smoking history & 2 & 0 \\
Brain metastasis & $7(17.5)$ & $2(5.7)$ \\
Eastern Cooperative Oncology Group status (ECOG), & $n(\%)$ \\
$\quad 0$ & $30(75)$ & $26(74.2)$ \\
1 & $3(7.5)$ & $7(20.1)$ \\
2 & $26(65)$ & $35(100)$ \\
Adenocarcinoma & $14(35)$ & 0 \\
Squamous cell carcinoma & $18(45)$ & $9(25.7)$ \\
First-line treatment & $22(55)$ & $26(74.2)$ \\
Second- and third-line treatment & $4(10)$ & $2(5.7)$ \\
Complete remission (CR) & $12(30)$ & $6(17.1)$ \\
Partial remission (PR) & $13(32.5)$ & $9(25.7)$ \\
Stable disease (SD) & $9(22.5)$ & $18(51.4)$ \\
Disease progression (PD) & &
\end{tabular}

2.3. Flow Cytometry. Prepare $100 \mu \mathrm{l}$ of sample solution, including $25 \mu \mathrm{l}$ of assay buffer, $25 \mu \mathrm{l}$ of each sample, $25 \mu \mathrm{l}$ of premixed magnetic beads, and $25 \mu \mathrm{l}$ of detection antibody (R 701001, Qingdao Raisecare Biological Technology Co. Ltd, China). Then, shake on a plate shaker (about $500 \mathrm{rpm}$ ) for 2 hours at room temperature. After that, $25 \mu \mathrm{l}$ of SA-PE was added to each test tube and shaken (approximately $500 \mathrm{rpm}$ ) for 30 minutes at room temperature. $500 \mu \mathrm{l}$ of $1 \mathrm{X}$ wash buffer was added to all test tubes and centrifuge for 5 minutes at 300-500 g. Remove the supernatant. $150 \mu \mathrm{l}$ of $1 \mathrm{X}$ wash buffer was added to all test tubes. Vortex for 30 seconds to resuspend the beads. All operations need to be protected from light. Read the sample on the flow cytometer (Beckman Coulter Epics XL Cytometer).

2.4. Statistical Analyses. The statistical analysis was performed by GraphPad Prism 8.2.1 software (GraphPad Software Inc., USA). The association between IL-5 and IFN- $\gamma$ levels and clinical response were analyzed by the nonparametric Wilcoxon matched-pairs ranked tests. The PFS data were obtained by standardized imaging assessment of all patients. The analysis of survival situation was followed up to January 31, 2020. Kaplan-Meier and the log rank test were used to plot the survival curve. $P<0.05$ was considered as statistically significant difference.

\section{Results}

3.1. Patient Population. From January 2017 to December 2019, a total of 75 consecutive patients received anti-PD1 therapy at Changzhou Tumor Hospital affiliated to Soochow University and met study inclusion criteria. These patients 


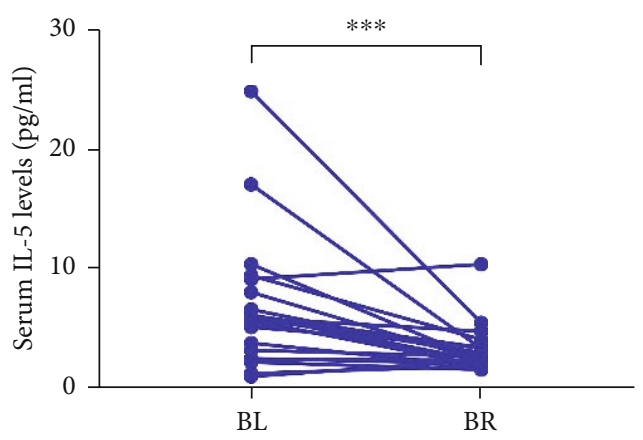

(a)

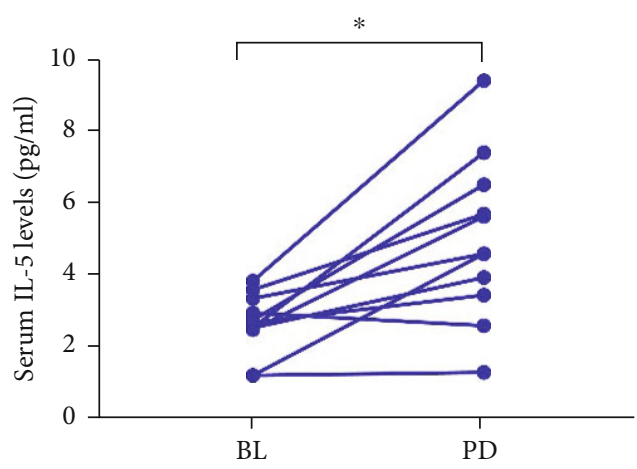

(c)

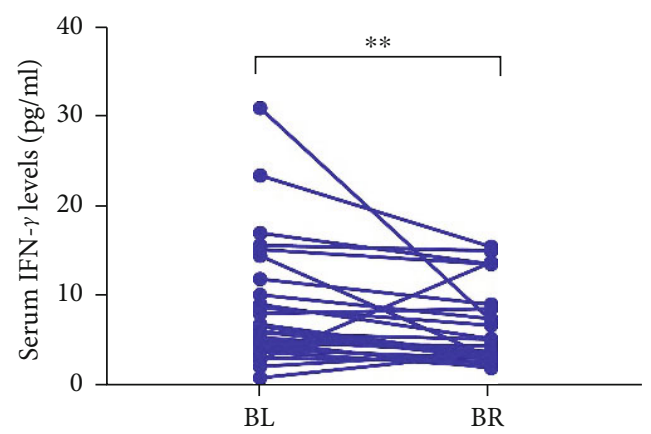

(b)

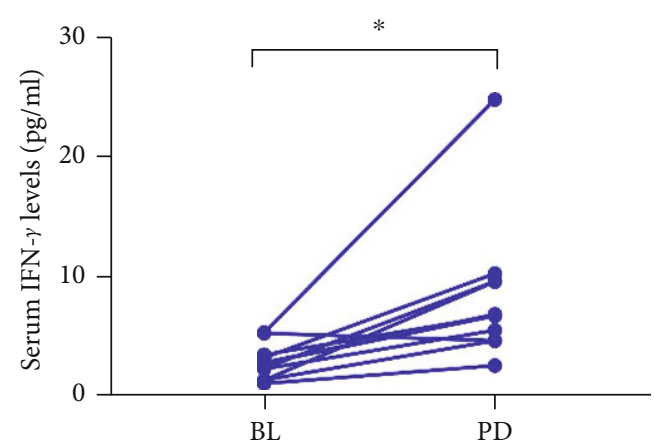

(d)

Figure 1: Serum IL-5 and IFN- $\gamma$ levels reflect clinical response to anti-PD-1 mAbs in NSCLC patients. Serum IL-5 and IFN- $\gamma$ levels were assessed at baseline (BL), best response (BR), and progressive disease (PD). (a) The median IL-5 levels at BR were lower than that at BL in the effective group; (b) the median IFN- $\gamma$ levels at BR were lower than that at BL in the effective group; (c) the median IL-5 and IFN- $\gamma$ levels at PD were higher than that at BL in the ineffective group; (d) the median IL-5 and IFN- $\gamma$ levels at PD were higher than that at BL in the ineffective group. Statistical difference between median IL- 5 and IFN- $\gamma$ levels in different time-points was made by nonparametric Wilcoxon matched-pairs ranked tests. ${ }^{*} P<0.05 ;{ }^{* *} P<0.01 ;{ }^{* * *} P<0.001$.

diagnosed with NSCLC (40/75) or GC (35/75) took $200 \mathrm{mg}$ anti-PD1 McAb every two or three weeks. Patients were followed up for at least 24 months. According to RECISTv1.1 standard, the DCR of patients diagnosed with NSCLC or GC was $72.5 \%$ and $48.5 \%$, respectively. Two NSCLC patients were identified as pseudoprogression (after a first treatment cycle, a more than $20 \%$ increase of the tumor size which was assessed as a partial response by imaging system).

\subsection{Serum IL-5 and IFN- $\gamma$ Levels Associated with Response to} Anti-PD-1 Treatment in Metastatic NSCLC Patients. Serum IL-5 and IFN- $\gamma$ levels were tested in 40 patients with NSCLC during anti-PD-1 mAb treatment. In the effective group $(\mathrm{CR}+\mathrm{PR}+\mathrm{SD})(n=29)$, the median IL-5 level at best response (BR) was $2.56 \mathrm{pg} / \mathrm{ml}$, which was significantly lower than before treatment $(6.50 \mathrm{pg} / \mathrm{ml})(P<0.001$, Figure $1(\mathrm{a}))$. Similarly, the median IFN- $\gamma$ level at BR was $6.27 \mathrm{pg} / \mathrm{ml}$, which was significantly lower than before treatment $(8.74 \mathrm{pg} / \mathrm{ml})$ $(P<0.001$, Figure 1(b)). In the ineffective group (PD) $(n=11)$, the median IL-5 and IFN- $\gamma$ levels increased significantly as the disease progressed (baseline $2.43 \mathrm{pg} / \mathrm{ml}$ (IL-5), $2.77 \mathrm{pg} / \mathrm{ml}$ (IFN- $\gamma$ ) versus PD 4.87 (IL-5), $8.24 \mathrm{pg} / \mathrm{ml}$ (IFN$\gamma), P<0.05, P<0.05$, respectively) (Figures $1(\mathrm{c})$ and $1(\mathrm{~d})$ ).

3.3. Serum IL-5 and IFN- $\gamma$ Levels Associated with Response to Anti-PD-1 Treatment in Metastatic GC Patients. We also detected IL- 5 and IFN- $\gamma$ levels in 35 patients with metastatic GC. In the effective group $(\mathrm{CR}+\mathrm{PR}+\mathrm{SD})(n=17)$, the median IL-5 level at BR was $3.78 \mathrm{pg} / \mathrm{ml}$, which was significantly lower than before treatment $(7.07 \mathrm{pg} / \mathrm{ml})(P<0.01$, Figure $2(\mathrm{a}))$. Similarly, the median IFN- $\gamma$ level at BR was $6.29 \mathrm{pg} / \mathrm{ml}$, which was significantly lower than before treatment (3.27 pg/ml) $(P<0.01$, Figure 2(b)). In the ineffective group $(n=18)$, the median IL-5 and IFN- $\gamma$ levels increased significantly as the disease progressed (baseline: $2.37 \mathrm{pg} / \mathrm{ml}$ (IL-5), $2.81 \mathrm{pg} / \mathrm{ml}($ IFN- $\gamma$ ) versus BR: $4.67 \mathrm{pg} / \mathrm{ml}$ (IL-5); $7.51 \mathrm{pg} / \mathrm{ml}$ (IFN- $\gamma) ; P<0.01, P<0.01$, respectively) (Figures 2(c) and 2(d)).

3.4. Predictive Value of Serum IL-5 and IFN- $\gamma$ Levels for Pseudoprogression. Serum IL-5 and IFN- $\gamma$ levels were detected in two patients with NSCLC who were diagnosed as pseudoprogression before treatment and at the time of a pseudoprogression diagnosis. Interestingly, although the imaging evaluation showed that the tumor lesions increased during pseudoprogression, serum IL-5 and IFN- $\gamma$ levels inversely decreased and remained below the baseline levels for a long time (Figures 3(a) and 3(b)).

3.5. Decreased Serum IL-5 and IFN- $\gamma$ Levels Correlated with Positive PFS in NSCLC Patients Treated with Anti-PD-1 $m A b s$. Kaplan-Meier plots progression-free survival (PFS) 


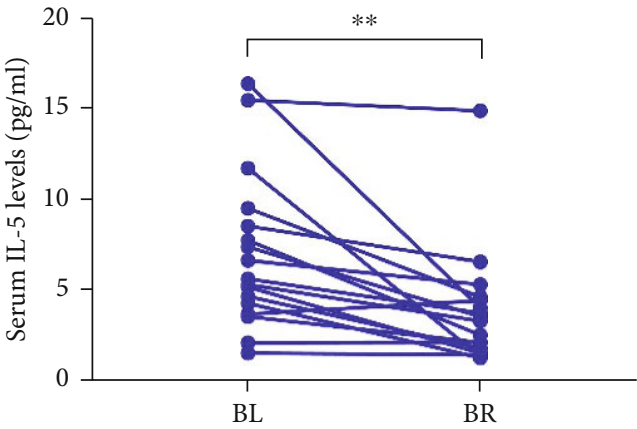

(a)

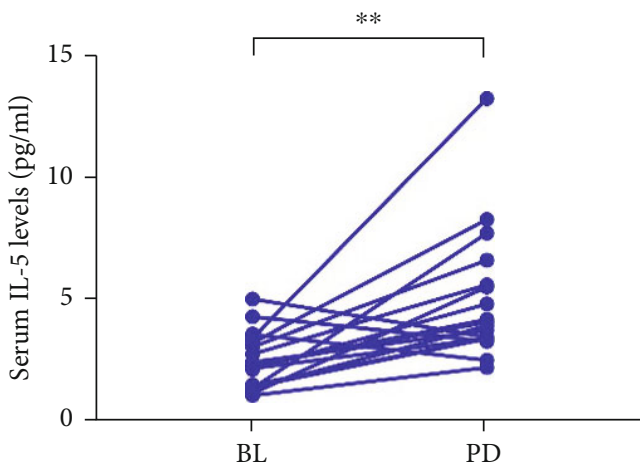

(c)

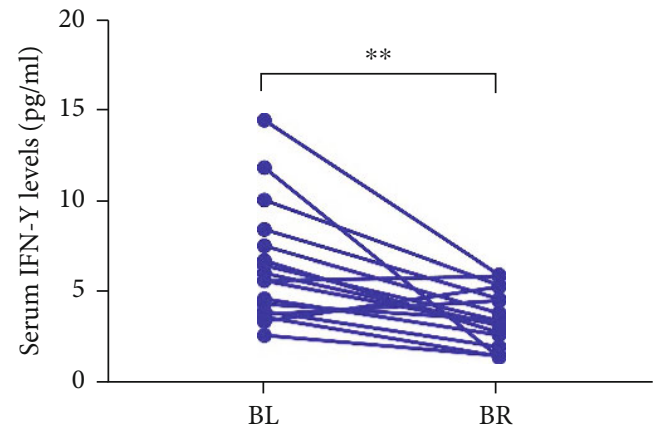

(b)

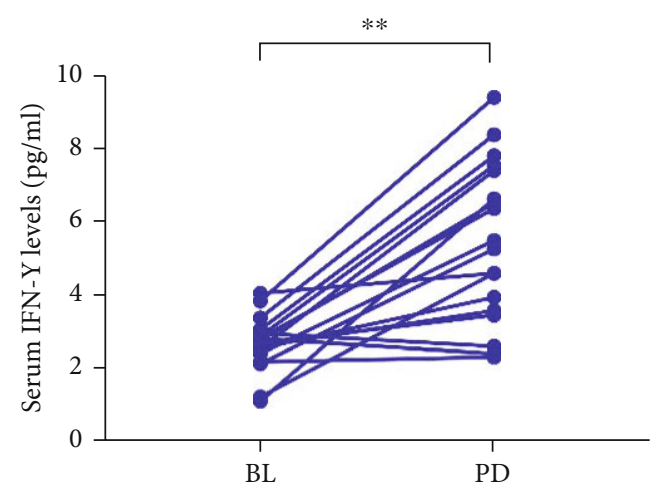

(d)

FIGURE 2: Serum IL-5 and IFN- $\gamma$ levels reflect clinical response to anti-PD-1 mAbs in metastatic GC patients. Serum IL-5 and IFN- $\gamma$ levels were assessed at BL, BR, and PD. (a) The median IL-5 levels at BR were lower than that at BL in the effective group; (b) the median IFN- $\gamma$ levels at BR were lower than that at BL in the effective group; (c) the median IL- 5 and IFN- $\gamma$ levels at PD were higher than that at BL in the ineffective group; (d) the median IL-5 and IFN- $\gamma$ levels at PD were higher than that at BL in the ineffective group. Statistical difference between median IL-5 and IFN- $\gamma$ levels in different time-points was made by nonparametric Wilcoxon matched-pairs ranked tests. ${ }^{*} P<$ $0.05,{ }^{* *} P<0.01,{ }^{* * *} P<0.001$.

stratified by early changes in the serum IL- 5 and IFN- $\gamma$ levels of NSCLC patients treated with anti-PD1 mAbs. It turned out that NSCLC patients whose serum IL- 5 and IFN- $\gamma$ initially decreased had longer progression-free survival than those who initially increased (median PFS (months): early decreases cohort: 11.2 (IL-5), 10.8 (IFN- $\gamma$ ) versus early increases cohort: 6.75 (IL-5), 7.3 (IFN- $\gamma$ )) (Figures 4(a) and 4(b)). However, such PFS difference was not observed in metastatic GC cohort.

\section{Discussion}

Immunocheckpoint inhibitors mainly target the inhibitory immune response in the tumor microenvironment, and it takes a long time to experience tumor shrinkage or regression. Studies have shown that the response to anti-PD-1 mAbs usually took 12 weeks and the reaction time even longer in some cases [11]. In addition, immunocheckpoint inhibitor therapy can lead to atypical tumor responses such as pseudoprogression which may occur in up to $15 \%$ of cases. Pseudoprogression refers to an unusual radiologic imaging of tumor response where a more than 20\% increase of target lesion size or the appearance of new lesions is not recognized as true disease progression [6]. It is crucial to identify biomarkers to predict the response of checkpoint blockades. The overexpression of PD-L1 on epithelial tumors is often treated with PD1/PD-L1 blocking antibodies. However, due to low prediction accuracy and dynamic changes, PD-L1 staining cannot be used to screen out patients sensitive to anti-PD-1 mAbs [12]. Tumorinfiltrating immune cells in the tumor microenvironment, gene analysis such as microsatellite instability, mismatchrepair deficiency, and tumor mutation burden may be important in predicting clinical benefits of PD-1/PD-L1 checkpoint blockades [13].

Peripheral blood collection is a less invasive, safe, convenient, and repeatable method for sample collection. Furthermore, peripheral blood can provide a systematic view of the host's immune status. In recent years, some biomarkers in the blood circulation have shown value in predicting the response of patients to immunotherapy, such as PD $-1^{+} \mathrm{CD} 8^{+} \mathrm{T}$ cells [14], $\mathrm{CD} 4^{+} \mathrm{T}$ cells [15], TCR repertoire $[16,17]$, cell-free DNA, circulating tumor cells, and cytokines [18].

In this study, we demonstrated that serum IL-5 and IFN$\gamma$ levels were correlated with the efficacy of anti-PD-1 treatment in metastatic GC and NSCLC patients. Moreover, we elucidated that early changes in serum IL-5 and IFN- $\gamma$ levels can predict the PFS in NSCLC patients treated with anti-PD-1 mAbs. 

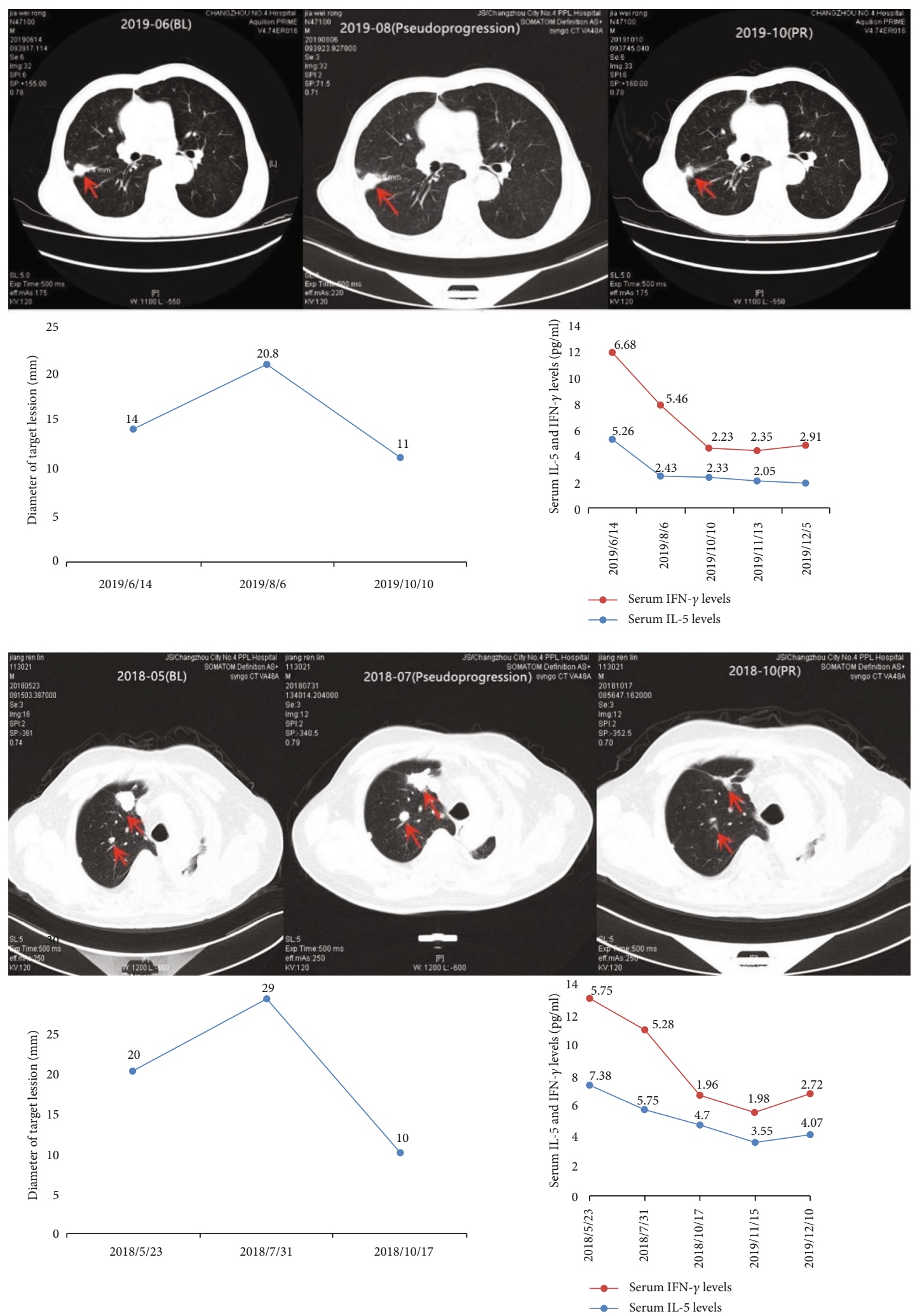

FIGURE 3: Serum IL-5 and IFN- $\gamma$ levels reflect pseudoprogression in 2 metastatic NSCLC patients during anti-PD-1 mAb treatment. Serum IL-5 and IFN- $\gamma$ levels were tested at baseline, at pseudoprogression diagnosis (first increase $>25 \%$ in tumor lesion size) and at subsequent imaging evaluations that fulfilled criteria of partial response (PR). Although imaging-evaluated increases in the lesion size during pseudoprogression, serum IL-5 and IFN- $\gamma$ levels decreased and remained below the baseline levels. 


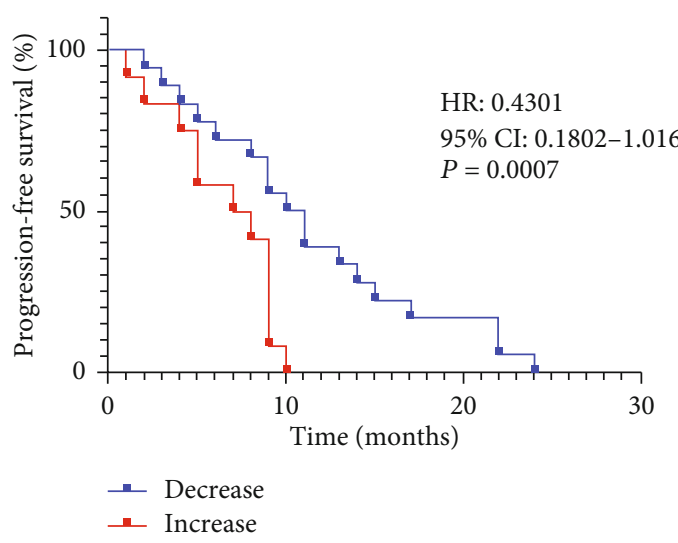

(a)

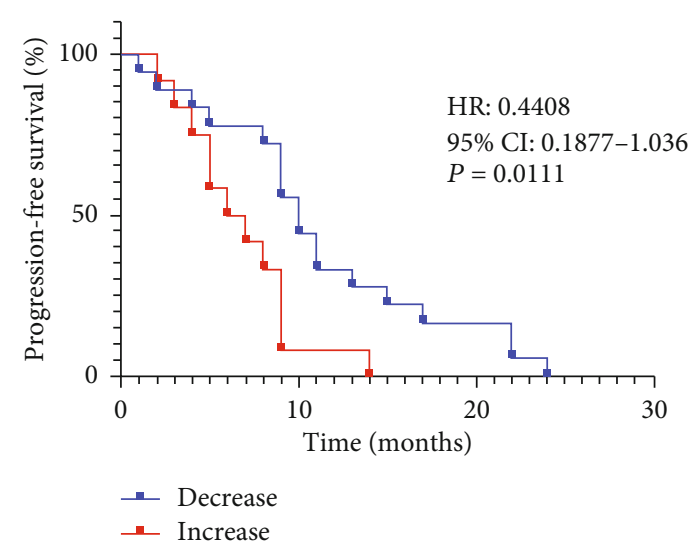

(b)

FIGURE 4: Serum IL-5 and IFN- $\gamma$ levels associated with progression-free survival in metastatic NSCLC patients treated with anti-PD-1 mAbs. Patients were stratified into two subgroups (decrease and increase) based on IL-5 or IFN- $\gamma$ levels at BL. Kaplan-Meier plots depict PFS differences between IL-5 (a) or IFN- $\gamma$ (b) levels. Data were analyzed by the log-rank (Mantel-Cox) test.

IL-5 and IFN- $\gamma$ levels also can be used to distinguish pseudoprogression from true disease progression. It appeared that lymphocyte infiltration is associated with tumor-related lesions when pseudoprogression occurs. It is hard to identify subcellular changers of tumor lesions by imaging technology. In our study, we for the first time found that serum IL- 5 and IFN- $\gamma$ levels can be useful biomarkers reflecting the changes in tumor areas. Moreover, we introduced two cases of pseudoprogression. They initially showed an increase in tumor lesion, and a relatively significant reduction was evaluated by imaging techniques. When tumor lesions increased evaluated by imaging methods, serum IL5 and IFN- $\gamma$ levels were lower than baseline level. These results suggested that serum IL-5 and IFN- $\gamma$ levels might become useful biomarkers to identify pseudoprogression. However, more pseudoprogresssion samples are needed to confirm this finding.

IL-5 is mainly produced by leukocytes including T cells, eoinophils, basophils, and natural helper cells [19]. Studies have found that innate IL-5-producing cells localized most abundantly in the lung and contributed to maintaining enough lung eosinophils. Given that eosinophils have proven to have antitumor activity, Ikutani et al. showed that innate IL-5-producing cells were increased in response to tumor invasion, and their regulation of eosinophils is critical to suppress tumor metastasis. Conversely, exogenous IL-5 treatment resulted in suppressed tumor metastasis and augmented eosinophil infiltration [10]. These newly identified innate IL-5-producing cells thus play a role in tumor surveillance through lung eosinophils and may contribute to development of novel immunotherapies for cancer. We found a close correlation between serum IL-5 levels and clinical efficacy of anti-PD-1 mAb treatment. This can be explained by the fact that when anti-PD-1 mAb treatment was effective, tumor burden and invasion range were subsequently reduced. Then, IL-5-producing cells are decreased by negative feedback, resulting in a decrease in IL-5 levels. This may also apply to the correlation between IFN- $\gamma$ level and clinical response to anti-PD-1 mAb treatment. Moreover, potential inflammatory conditions should be considered in our study. In this regard, we have detected inflammatory factors including IL-1, IL-6, and TNF- $\alpha$ to help identify whether the increase of IL- 5 and IFN- $\gamma$ levels was caused by tumor progression or the concurrent inflammatory conditions. Results showed that none of these common inflammatory factors was associated with the clinical response to anti-PD-1 $\mathrm{mAb}$ treatment.

There was no correlation between pretreatment of IL-5 and IFN- $\gamma$ levels and the efficacy of anti-PD-1 mAbs. It may be because the therapeutic responses to anti-PD-1 $\mathrm{mAb}$ treatment are complicated and involve multiple immune processes $[20,21]$. In addition, the therapeutic response to anti-PD-1 mAbs has been suggested to be a critical state transition process of a complex system, which is hard to predict long-term in advance [22].

\section{Conclusion}

We found that serum IL-5 and IFN- $\gamma$ levels were closely associated with tumor burden in metastatic NSCLC and GC patients receiving anti-PD-1 mAbs, so their expression levels can assess the efficacy of anti-PD-1 mAb treatment. Moreover, early changes of their levels correlated with progression-free survival in patients with metastatic NSCLC patients. Finally, IL-5 and IFN- $\gamma$ levels had a better predictive value than imaging techniques in a small number of patients diagnosed with pseudoprogression. Metastatic NSCLC patients with initially decreased IL-5 and IFN- $\gamma$ levels obtained positively clinical outcome and long PFS with anti-PD-1therapy. Our study provides effective biomarkers for the evaluation of efficacy and prognosis of anti-PD-1 $\mathrm{mAb}$ treatment; however, the number of patients in our study is limited, and further prospective validation including multiple types of cancers is required.

\section{Data Availability}

The data used in the study are available from the corresponding author upon request. 


\section{Conflicts of Interest}

The authors declare that they have no conflict of interests.

\section{Acknowledgments}

This study was supported by grants from the Changzhou Health and Family Planning Commission. Applied Basic Research Program of Changzhou [CJ20200103].

\section{References}

[1] F. Hirano, K. Kaneko, H. Tamura et al., "Blockade of B7-H1 and PD-1 by monoclonal antibodies potentiates cancer therapeutic immunity," Cancer Research, vol. 65, no. 3, pp. 10891096, 2005.

[2] G. J. Freeman, A. J. Long, Y. Iwai et al., "Engagement of the PD-1 immunoinhibitory receptor by a novel B7 family member leads to negative regulation of lymphocyte activation," The Journal of Experimental Medicine, vol. 192, no. 7, pp. 1027-1034, 2000.

[3] G. P. Dunn, A. T. Bruce, H. Ikeda, L. J. Old, and R. D. Schreiber, "Cancer immunoediting: from immunosurveillance to tumor escape," Nature Immunology, vol. 3, no. 11, pp. 991998, 2002.

[4] L. Chen and X. Han, "Anti-PD-1/PD-L1 therapy of human cancer: past, present, and future," The Journal of Clinical Investigation, vol. 125, no. 9, pp. 3384-3391, 2015.

[5] H. Iwai, J. Hamanishi, K. Chamoto, and T. Honjo, "Cancer immunotherapies targeting the PD-1 signaling pathway," Journal of Biomedical Science, vol. 24, no. 1, p. 26, 2017.

[6] F. S. Hodi, W.-J. Hwu, R. Kefford et al., "Evaluation of immune-related response criteria and RECIST v1.1 in patients with advanced melanoma treated with pembrolizumab," Journal of Clinical Oncology, vol. 34, no. 13, pp. 1510-1517, 2016.

[7] M. Moreno, J. W. Molling, S. von Mensdorff-Pouilly et al., "IFN-gamma-producing human invariant NKT cells promote tumor-associated antigen-specific cytotoxic T cell responses," Journal of Immunology, vol. 181, no. 4, pp. 2446-2454, 2008.

[8] B. Silva-Santos, K. Serre, and H. Norell, " $\gamma \delta$ T cells in cancer," Nature Reviews. Immunology, vol. 15, no. 11, pp. 683-691, 2015.

[9] K. Schroder, P. J. Hertzog, T. Ravasi, and D. A. Hume, "Interferon-gamma: an overview of signals, mechanisms and functions," Journal of Leukocyte Biology, vol. 75, no. 2, pp. 163189, 2004.

[10] M. Ikutani, T. Yanagibashi, M. Ogasawara et al., "Identification of innate IL-5-producing cells and their role in lung eosinophil regulation and antitumor immunity," Journal of Immunology, vol. 188, no. 2, pp. 703-713, 2012.

[11] J. D. Wolchok, A. Hoos, S. O'Day et al., "Guidelines for the evaluation of immune therapy activity in solid tumors: immune-related response criteria," Clinical Cancer Research, vol. 15, no. 23, pp. 7412-7420, 2009.

[12] S. P. Patel and R. Kurzrock, "PD-L1 expression as a predictive biomarker in cancer immunotherapy," Molecular Cancer Therapeutics, vol. 14, no. 4, pp. 847-856, 2015.

[13] X. Meng, Z. Huang, F. Teng, L. Xing, and J. Yu, "Predictive biomarkers in PD-1/PD-L1 checkpoint blockade immunotherapy," Cancer Treatment Reviews, vol. 41, no. 10, pp. 868876, 2015.
[14] P. C. Tumeh, C. L. Harview, J. H. Yearley et al., "PD-1 blockade induces responses by inhibiting adaptive immune resistance," Nature, vol. 515, no. 7528, pp. 568-571, 2014.

[15] R. Zappasodi, S. Budhu, M. D. Hellmann et al., "Non-conventional Inhibitory CD $4^{+}$Foxp $3^{-} \mathrm{PD}-1^{\text {hi }} \mathrm{T}$ Cells as a Biomarker of Immune Checkpoint Blockade Activity," Cancer Cell, vol. 33, no. 6, pp. 1017-1032.e7, 2018.

[16] A. C. Hopkins, M. Yarchoan, J. N. Durham et al., "T cell receptor repertoire features associated with survival in immunotherapy-treated pancreatic ductal adenocarcinoma," JCI Insight, vol. 3, no. 13, article e122092, 2018.

[17] J. Han, J. Duan, H. Bai et al., “TCR repertoire diversity of peripheral $\mathrm{PD}-1+\mathrm{CD} 8+\mathrm{T}$ cells predicts clinical outcomes after immunotherapy in patients with non-small cell lung cancer," Cancer Immunology Research, vol. 8, no. 1, pp. 146-154, 2020.

[18] M. F. Sanmamed, J. L. Perez-Gracia, K. A. Schalper et al., "Changes in serum interleukin-8 (IL-8) levels reflect and predict response to anti-PD-1 treatment in melanoma and nonsmall-cell lung cancer patients," Annals of Oncology, vol. 28, no. 8, pp. 1988-1995, 2017.

[19] K. Takatsu, R. Dickason, and D. Huston, Interleukin-5, Growth Factors and Cytokines in Health and Disease, JAI Press Ltd., London, 1997.

[20] C. U. Blank, J. B. Haanen, A. Ribas, and T. N. Schumacher, "Cancer immunology. The "cancer immunogram"," Science, vol. 352, no. 6286, pp. 658-660, 2016.

[21] D. S. Chen and I. Mellman, "Oncology meets immunology: the cancer-immunity cycle," Immunity, vol. 39, no. 1, pp. 1-10, 2013.

[22] W. J. Lesterhuis, A. Bosco, M. J. Millward, M. Small, A. K. Nowak, and R. A. Lake, "Dynamic versus static biomarkers in cancer immune checkpoint blockade: unravelling complexity," Nature Reviews. Drug Discovery, vol. 16, no. 4, pp. 264272, 2017. 